Research Article

\title{
Some Properties of Double Roman Domination
}

\author{
Hong Yang $(\mathbb{D})$ and Xiaoqing Zhou \\ School of Information Science and Engineering, Chengdu University, Chengdu 610106, China \\ Correspondence should be addressed to Hong Yang; yanghong01@cdu.edu.cn
}

Received 22 May 2020; Accepted 18 July 2020; Published 14 August 2020

Academic Editor: Juan L. G. Guirao

Copyright (C) 2020 Hong Yang and Xiaoqing Zhou. This is an open access article distributed under the Creative Commons Attribution License, which permits unrestricted use, distribution, and reproduction in any medium, provided the original work is properly cited.

\begin{abstract}
A double Roman dominating function on a graph $G$ is a function $f: V(G) \longrightarrow\{0,1,2,3\}$ satisfying the conditions that every vertex $u$ for which $f(u)=0$ is adjacent to at least one vertex $v$ for which $f(v)=3$ or two vertices $v_{1}$ and $v_{2}$ for which $f\left(v_{1}\right)=f\left(v_{2}\right)=2$ and every vertex $u$ for which $f(u)=1$ is adjacent to at least one vertex $v$ for which $f(v) \geq 2$. The weight of a double Roman dominating function $f$ is the value $f(V)=\sum_{u \in V} f(u)$. The minimum weight of a double Roman dominating function on a graph $G$ is called the double Roman domination number $\gamma_{d R}(G)$ of $G$. A graph with $\gamma_{d R}(G)=3 c(G)$ is called a double Roman graph. In this paper, we study properties of double Roman domination in graphs. Moreover, we find a class of double Roman graphs and give characterizations of trees with $\gamma_{d R}(T)=c_{R}(T)+k$ for $k=1,2$.
\end{abstract}

\section{Introduction}

In this paper, we shall only consider graphs without multiple edges or loops. Let $G$ be a graph, $v \in V(G)$, and the neighborhood of $v$ in $G$ is denoted by $N(v)$. That is to say, $N(v)=\{u \mid u v \in E(G), u \in V(G)\}$. The closed neighborhood $N[v]$ of $v$ in $G$ is defined as $N[v]=\{v\} \cup N(v)$. The complementary graph of $G$ is denoted by $\bar{G}$. A vertex of degree one is called a leaf. A graph is trivial if it has a single vertex. The degree of a vertex $v$ is denoted by $d(v)$, i.e., $d(v)=|N(v)|$. Denote by $K_{n}, P_{n}$, and $C_{n}$ the complete graph, path, and cycle on $n$ vertices, respectively. The maximum degree and the minimum degree of a graph are denoted by $\Delta(G)$ and $\delta(G)$, respectively. For a set $S \subseteq V(G)$, the graph induced by $S$ is denoted by $G[S]$. Let $e \in E(G)$, and we denote by $G / e$ the graph obtained from $G$ by contracting the edge $e$. For an edge $e \in E(G)$, we denote by $G-e$ the graph obtained from $G$ by deleting $e$.

A subset $D$ of the vertex set of a graph $G$ is a dominating set if every vertex not in $D$ has at least one neighbour in $D$. The domination number $\gamma(G)$ is the minimum cardinality of a dominating set of $G$.

The domination and its variations of graphs have attracted considerable attention $[1,2]$. Many varieties of dominating sets are listed in the book Fundamentals of Domination in Graphs [3]. However, Roman domination and double Roman domination are not listed in this book. Roman domination and double Roman domination appear to be a new variety of interest [4-11].

A Roman dominating function (RDF) of a graph $G$ is a function $f: V(G) \longrightarrow\{0,1,2\}$ such that every vertex $u$ for which $f(u)=0$ is adjacent to at least one vertex $v$ for which $f(v)=2$. The weight $w(f)$ of a Roman dominating function $f$ is the value $w(f)=\sum_{u \in V(G)} f(u)$. The minimum weight of a Roman dominating function on a graph $G$ is called the Roman domination number $\gamma_{R}(G)$ of $G$. An RDF $f$ of $G$ with $w(f)=\gamma_{R}(G)$ is called a $\gamma_{R}(G)$ function.

A double Roman dominating function (DRDF) on a graph $G$ is a function $f: V(G) \longrightarrow\{0,1,2,3\}$ satisfying the condition that every vertex $u$ for which $f(u)=0$ is adjacent to at least one vertex $v$ for which $f(v)=3$ or two vertices $v_{1}$ and $v_{2}$ for which $f\left(v_{1}\right)=f\left(v_{2}\right)=2$ and every vertex $u$ for which $f(u)=1$ is adjacent to at least one vertex $v$ for which $f(v) \geq 2$. The weight $w(f)$ of a double Roman dominating function $f$ is the value $w(f)=\sum_{u \in V(G)} f(u)$. The minimum weight of a double Roman dominating function on a graph $G$ is called the double Roman domination number $\gamma_{d R}(G)$ of G. A DRDF $f$ of $G$ with $w(f)=\gamma_{d R}(G)$ is called a $\gamma_{d R}(G)$ function. We denote by $w_{S}(f)$ the weight of a double Roman dominating function $f$ in $S \subseteq V(G)$, i.e., $w_{S}(f)=\sum_{x \in S} f(x)$. 
Beeler et al. [12] initiated the study of the double Roman domination in graphs. They showed that $2 \gamma(G) \leq$ $\gamma_{d R}(G) \leq 3 \gamma(G)$ and defined a graph $G$ to be double Roman if $\gamma_{d R}(G)=3 \gamma(G)$. Moreover, they suggest to find double Roman graphs.

In this paper, we study properties of double Roman domination in graphs and show that the double Roman domination problem is NP-complete for bipartite graphs. Moreover, we find a class of double Roman graphs and give characterizations of trees with $\gamma_{d R}(T)=\gamma_{R}(T)+k$ for $k=1,2$.

\section{Properties of Double Roman Domination}

Proposition 1 (see [12]). In a double Roman dominating function of weight $\gamma_{d R}(G)$, no vertex needs to be assigned the value 1 .

By Proposition 1, when we consider a $\gamma_{d R}(G)$ function, we assume no vertex has been assigned the value 1 .

\section{Proposition 2 (see [12])}

(i) Let $G$ be a graph and $f=\left(V_{0}, V_{1}, V_{2}\right)$ be a $\gamma_{R}(G)$ function. Then, $\gamma_{d R}(G) \leq 2\left|V_{1}\right|+3\left|V_{2}\right|$.

(ii) For any graph $G, \gamma_{d R}(G) \leq 2 \gamma_{R}(G)$ with equality if and only if $G=\overline{K_{n}}$.

Proposition 3 (see [12])

(i) For every graph $G, \gamma_{R}(G)<\gamma_{d R}(G)$.

(ii) If $f=\left(V_{0}, \varnothing, V_{2}, V_{3}\right)$ is any $\gamma_{d R}(G)$ function, then $\gamma_{R}(G) \leq 2\left(\left|V_{2}\right|+\left|V_{3}\right|\right)=\gamma_{d R}(G)-\left|V_{3}\right|$.

The following result is immediate.

Proposition 4. For any graph $G, \quad \gamma_{d R}(G) \geq(3|V(G)| /$ $\Delta(G)+1)$.

Proof. The desired inequality obviously holds if $\Delta(G) \leq 1$. In order to prove the proposition for $\Delta(G) \geq 2$, we introduce the discharging approach. Let $f=\left(V_{0}, \varnothing, V_{2}, V_{3}\right)$ be a $\gamma_{d R}(G)$ function. The initial charge of every vertex $v \in V(G)$ is set to be $s(v)=f(v)$. We apply the discharging procedure defined by applying the following rule.

For each vertex $v \in V_{3}$, we send $3 /(d(v)+1)$ charge to each adjacent vertex in $V_{0}$. Then, the final charge of $v$ is satisfying with $s(v)=(3 /(d(v)+1)) \geq(3 /(\Delta(G)+1))$.

For each vertex $v \in V_{2}$, we send $(2 /(d(v)+1))-$ $(3 /(d(v)(\Delta(G)+1)))=((2 \Delta(G)-1) /(d(v)(\Delta(G)+1))) \geq$ $((2 \Delta(G)-1) /(\Delta(G)(\Delta(G)+1)))$ charge to each adjacent vertex in $V_{0}$. Then, the final charge of $v$ is satisfying with $s(v)=(3 /(d(v)+1)) \geq(3 /(\Delta(G)+1))$.

For each vertex $v \in V_{0}$, by the definition of double Roman domination, $v$ has a neighbor assigned 3 or two vertices $u_{1}$ and $u_{2}$ assigned 2 . Due to the discharging rule above, if $v$ has a neighbor $u$ assigned 3, $v$ receives charge from $u$. We have $s(v) \geq(3 /(\Delta(G)+1))$.
If $v$ has two vertices $u_{1}$ and $u_{2}$ assigned 2, $v$ receives charge from $u_{1}$ and $u_{2}$. We have $s(v) \geq((4 \Delta(G)-2) /$ $(\Delta(G)(\Delta(G)+1))) \geq(3 /(\Delta(G)+1))$. Thus, $\quad \gamma_{d R}(G)=$ $\sum_{v \in V(G)} f(v)=\sum_{v \in V(G)} s(v) \geq((3|V(G)|) /(\Delta(G)+1))$. The proof is complete.

Proposition 5. Let $G$ be a graph. If $\gamma_{R}(G)+1=\gamma_{d R}(G)$ and $f=\left(V_{0}, \varnothing, V_{2}, V_{3}\right)$ is a $\gamma_{d R}(G)$ function,

(i) then $\left|V_{3}\right| \leq 1$

(ii) if $\left|V_{3}\right|=1$, then $\left|V_{2}\right|=0$ and there exists a vertex $v$ with degree $|V(G)|-1$.

Proof

(i) By Proposition 3, we have $\gamma_{R}(G) \leq \gamma_{d R}(G)-\left|V_{3}\right|=$ $\gamma_{R}(G)+1-\left|V_{3}\right|$. So we have $\left|V_{3}\right| \leq 1$.

(ii) If $\left|V_{3}\right|=1$, let $V_{3}=\{v\}$ and $H=G\left[V(G)-N\left[V_{3}\right]\right]$. We have the following claim.

Claim 1. $H$ is empty.

Proof. Otherwise, any vertex in $H$ assigned with 0 has at least two vertices assigned with 2 . Let $w$ be a vertex in $H$ assigned with 2 , we consider a function $f^{\prime}$ with $f^{\prime}(w)=1$, $f^{\prime}(v)=2$, and $f^{\prime}(x)=f(x)$ for any $x \in V(G)-\{v, w\}$. Then, $f^{\prime}$ is an RDF of $G$ with weight $\gamma_{d R}(G)-2$. So $\gamma_{R}(G) \leq \gamma_{d R}(G)-2$, a contradiction.

Since $H$ is empty, we have $v$ as a vertex with degree $|V(G)|-1$. Now, we have $\gamma_{d R}(G)=3$ and $\gamma_{R}(G)=2$, and so the result holds.

Theorem 1. For every graph $G$ on $n$ vertices without isolated vertex, $\gamma_{d R}(G) \leq 3 n-(3 n /(2(1+\delta(G)))) e^{1 / \delta(G)}$.

Proof. Clearly, we have $\delta(G) \geq 1$. We select a subset $S$ of $V(G)$, where each vertex is selected with probability $p$ independently. Let $T=V(G)-N[S]$, we consider a function $f: V(G) \longrightarrow\{0,2,3\}$ with $f(x)=3$ for $x \in S, f(x)=2$ for $x \in T$, and $f(x)=0$ for other vertex $x$. Then, $f$ is a DRDF of $G$. We have $\gamma_{d R}(G) \leq 3|S|+2|T|$. When we consider the expectation, we also have $\gamma_{d R}(G) \leq E[3|S|+2|T|]=$ $3 E[|S|]+2 E[|T|]$. First, it is clear that $E[|S|]=n p$. For each vertex with degree $d(x)$, if neither $x$ nor any neighbor is selected, then $x \in T$. So we have $P(x \in T)=(1-p)^{1+d(x)}$, and thus, $E[|T|] \leq n(1-p)^{1+\delta(G)}$. Consequently, $\gamma_{d R}(G) \leq$ $3 n p+2 n(1-p)^{1+\delta(G)}$. Let $F(p)=3 n p+2 n(1-p)^{1+\delta(G)}$. By $F(p)=3 n p-2 n(1+\delta(G))(1-p)^{\delta(G)}=0$, the maximum of $F$ is given by $F_{\max }=3 n-(3 n /(2(1+\delta(G)))) e^{1 / \delta(G)}$ at $p=1-(3 /(2(1+\delta(G)))) e^{1 / \delta(G)} \in(0,1)$. Thus, $\gamma_{d R}(G) \leq$ $3 n-(3 n /(2(1+\delta(G)))) e^{1 / \delta(G)}$.

If $G$ is a graph with some isolated vertices, then $\delta(G)=0$. Let $W$ be the set of isolated vertices of $G$ and let $G^{\prime}=G-W$. Therefore, $\delta\left(G^{\prime}\right) \geq 1$. Because all isolated vertices must be assigned 3 , it is easy to prove that $\gamma_{d R}(G) \leq 3 n-$ $\left(3 n^{\prime} /\left(2\left(1+\delta\left(G^{\prime}\right)\right)\right)\right) e^{1 / \delta\left(G^{\prime}\right)}$, where $n^{\prime}=\left|V\left(G^{\prime}\right)\right|$. 
Proposition 6. If $G$ is a connected graph of order $n$, then $\gamma_{d R}(G)+1=2 \gamma_{R}(G)$ if and only if there exists a vertex $v$ of degree $n+1-\left(\left(\gamma_{d R}(G)+1\right) / 2\right)$ in $G$.

Proof

$(\Rightarrow)$ Let $f=\left(V_{0}, V_{1}, V_{2}\right)$ be a $\gamma_{R}(G)$ function with minimum $\left|V_{1}\right|$. Then, we have $V_{1}$ being independent. Together with Proposition 2, we have $\gamma_{d R}(G) \leq 2\left|V_{1}\right|+$ $3\left|V_{2}\right| \leq 2\left|V_{1}\right|+4\left|V_{2}\right|=2 \gamma_{R}(G)=\gamma_{d R}(G)+1$. Then, we have $\left|V_{2}\right| \leq 1$. If $\left|V_{2}\right|=0$, we have $\left|V_{0}\right|=0$, and thus $\gamma_{R}(G)=n=\left|V_{1}\right|$. Since $V_{1}$ is independent, it is impossible. If $\left|V_{2}\right|=1$, we have $\gamma_{d R}(G)=2\left|V_{1}\right|+3\left|V_{2}\right|$ $<2\left|V_{1}\right|+4\left|V_{2}\right|=2 \gamma_{R}(G)=\gamma_{d R}(G)+1$. Let $V_{2}=\{v\}$, $V_{0}=N(v)$, and $V_{1}=V(G)-V_{0}-V_{2}$. Then, we have $\gamma_{R}(G)=n-1-d(v)+2$ and so $d(v)=n+1-\gamma_{R}(G)$ $=n+1-\left(\left(\gamma_{d R}(G)+1\right) / 2\right)$.

$(\Leftarrow)$ By Proposition 2 (ii), we have $\gamma_{d R}(G)+1 \leq 2 \gamma_{R}(G)$ for a connected graph $G$. Assume $G$ contains a vertex $v$ of degree $n+1-\left(\gamma_{d R}(G)+1\right) / 2$ in $G$. Let $V_{2}=\{v\}$, $V_{0}=N(v), \quad$ and $V_{1}=V(G)-V_{0}-V_{2}$. Then, $f=\left(V_{0}, V_{1}, V_{2}\right)$ is a $\gamma_{R}(G)$ function and so $\gamma_{R}(G) \leq\left|V_{1}\right|+2\left|V_{2}\right|=\left(\gamma_{d R}(G)+1\right) / 2$. Hence, $\gamma_{d R}(G)+1 \geq 2 \gamma_{R}(G)$.

Let $\mathscr{F}$ be the family of connected graphs $G$ such that for any $\gamma_{d R}(G)$ function $f=\left(V_{0}, \varnothing, V_{2}, V_{3}\right)$, we have $\left|V_{3}\right|=0$.

Proposition 7. Let $G \in \mathscr{F}$, then

(i) G contains no strong support vertex

(ii) if $f(u)=f(v)=2$ for an edge $u v \in E(G)$, then $G-$ $e \in \mathscr{F}$ and $G / e \in \mathscr{F}$.

Proof

(i) Suppose $v$ be a strong support vertex, then there exist two leaves $x, y \in N(v)$. Since $f(v) \neq 3$, we have $f(x)=f(y)=2$. Now consider the function $f^{\prime}$ with $f^{\prime}(z)=0$ for any $z \in L(v), f^{\prime}(v)=3$, and $f^{\prime}(z)=f(z)$ for any $z \in V(G) \backslash L[v]$. Then, $f^{\prime}$ is a DRDF of $G$ with fewer weight than $f$, a contradiction.

(ii) If $f(u)=f(v)=2$ for an edge $u v \in E(G)$, we have $f$ as also a DRDF of $G-e$. So $\gamma_{d R}(G-e) \leq w(f)=\gamma_{d r}(G)$. Since for any graph $G$, we have $\quad \gamma_{d R}(G-e) \geq \gamma_{d R}(G)$, so $\gamma_{d R}(G-e)=\gamma_{d R}(G)$. Suppose to the contrary that there exists a $\gamma_{d R}(G-e)$ function such that $f(v)=3$ for a vertex $v \in V(G-e)$. Then, $f$ is also a $\gamma_{d R}(G)$ function, contradicting with $\left|V_{3}\right|=0$. For the graph $G / e$, the proof is similar.

Lemma 1. Let $G$ be a graph on $n \geq 4$ vertices, then $\gamma_{d R}(G)=$ 4 if and only if $G$ contains a complete bipartite graph $K_{2, n-2}$ as a subgraph and $\Delta(G) \leq n-2$.
Proof

$(\Rightarrow)$ If $\gamma_{d R}(G)=4$, then no vertex is assigned with 3 and thus we have two vertices $v$ and $w$ assigned with 2 and the others 0 . Also, each vertex 0 must be adjacent to both $v$ and $w$. Therefore, $G$ contains a complete bipartite graph $K_{2, n-2}$ as a subgraph. Since $\gamma_{d R}(G)>3$, we have $\Delta(G) \leq n-2$.

$(\Leftarrow)$ If $G$ contains a complete bipartite graph $K_{2, n-2}$ with partitions $X, Y(|X|=2,|Y|=n-2)$ as a subgraph and $\Delta(G) \leq n-2$. Then, let $f(x)=2$ for any $x \in X$ and $f(x)=0$ for $x \in Y$. Then, $f$ is a DRDF of $G$ and so $\gamma_{d R}(G) \leq 4$. Since $G$ contains no vertex with degree $|V(G)|-1$, we have $\gamma_{d R}(G) \geq 4$.

Note that $\Delta(G) \geq n-2$ if $G$ contains a complete bipartite graph $K_{2, n-2}$ as a subgraph. Thus, $\Delta(G) \leq n-2$ can be replaced with $\Delta(G)=n-2$ in the lemma.

Theorem 2. Let $G$ be a graph on $n \geq 3$ vertices, then $8 \leq \gamma_{d R}(G)+\gamma_{d R}(\bar{G}) \leq 2 n+3$. Furthermore, equality holds in the upper bound if $G$ or $\bar{G}$ is $K_{n}$.

Proof. If $G$ is a graph on $n \geq 3$ vertices, we have $\gamma_{d R}(G) \geq 3$, and if $\gamma_{d R}(G)=3$, then $G$ has a vertex with degree $n-1$. But its complement is neither a star nor a graph $G$ with $\gamma_{d R}(G)=$ 4 (see the graph stated in Lemma 1). So we have $\gamma_{d R}(\bar{G}) \geq 5$ and thus, $\gamma_{d R}(G)+\gamma_{d R}(\bar{G}) \geq 8$. If $G$ is a star, then $\gamma_{d R}(\bar{G})=5$ and so the lower bound is attainable.

Let $v$ be a vertex with maximum degree $\Delta(G)$; consider a function $f$ with $f(v)=3, f(x)=0$ for any $x \in N(v)$, and $f(x)=2$ for $x \in V(G)-N[x]$. Then, $f$ is a DRDF of $G$ and so $\gamma_{d R}(G) \leq w(f)=2 n-2 \Delta(G)+1$. Since $\Delta(\bar{G})+\delta(G)=$ $n-1$, we have $\gamma_{d R}(\bar{G}) \leq 2 n-2 \Delta(\bar{G})+1=2 \delta(G)+3$. Therefore, $\quad \gamma_{d R}(G)+\gamma_{d R}(\bar{G}) \leq 2 n+4-2 \Delta(G)+2 \delta(G) \leq$ $2 n+4$. It can be seen that if $\gamma_{d R}(G)+\gamma_{d R}(\bar{G})=2 n+4$, then $\Delta(G)=\delta(G)$. Hence, $G$ is $k$-regular for some $k$. By symmetry, we may assume that $k \leq(n-1) / 2$. Then, if $\gamma_{d R}(G)+\gamma_{d R}(\bar{G})=2 n+4$, we have $\gamma_{d R}(G)=2 n-2 k+1$ and $\gamma_{d R}(\bar{G})=2 k+3$. Let $v \in V(G)$. If $|N(u) \cap N(v)| \leq k-2$ for some $u \in V(G)-N[v]$, then $f=(N(v) \cup N(u)$, $\varnothing, V(G)-N[u]-N[v],\{u, v\})$ is a DRDF of $G$ with fewer weight than $2 n-2 k+1$, a contradiction. Therefore, each vertex not in $N[v]$ has at least $k-1$ neighbors in $N(v)$. Analogously, each vertex in $N[v]$ has at most 2 neighbors outside $N[v]$. Then, we have $(k-1)(n-k-1) \leq 2 k$. Since $k \leq(n-1) / 2$, we have $k \leq 2+2 /(k-1)$. If $k=3$, then $n=7$. This is impossible. If $k=2$, then $n \in\{5,6,7\}$. If $G=C_{5}$, we have $\gamma_{d R}(G)=\gamma_{d R}(\bar{G})=6$, and so $\gamma_{d R}(G)+\gamma_{d R}(\bar{G}) \neq$ $2 n+4$, a contradiction. If $G=C_{6}$, we have $\gamma_{d R}(G)=$ $\gamma_{d R}(\bar{G})=6$, and so $\gamma_{d R}(G)+\gamma_{d R}(\bar{G}) \neq 2 n+4$, a contradiction. If $G=C_{7}$, we have $\gamma_{d R}(G)=8$ and $\gamma_{d R}(\bar{G})=6$, and so $\gamma_{d R}(G)+\gamma_{d R}(\bar{G}) \neq 2 n+4$, a contradiction. If $k=1$, then $G=(n / 2) K_{2}$, and we have $\gamma_{d R}(G)=3 n / 2$ and $\gamma_{d R}(\bar{G})=4$, and so $\gamma_{d R}(G)+\gamma_{d R}(\bar{G}) \neq 2 n+4$, a contradiction. Therefore, we conclude that $8 \leq \gamma_{d R}(G)+\gamma_{d R}(\bar{G}) \leq 2 n+3$. If $G=K_{n}$, then $\gamma_{d R}(G)=3$ and $\gamma_{d R}(\bar{G})=2 n$ and thus the upper bound is attainable. 


\section{Some Double Roman Graphs}

The Cartesian product of graphs $G$ and $H$ is the graph $G \square H$ with vertex set $G \times H$ and $\left(x_{1}, x_{2}\right)\left(y_{1}, y_{2}\right) \in E(G \square H)$ whenever $x_{1} y_{1} \in E(G)$ and $x_{2}=y_{2}$, or $x_{2} y_{2} \in E(H)$ and $x_{1}=y_{1}$. The Cartesian product is commutative and associative, having the trivial graph as a unit (cf. [13]).

Let $f$ be a double Roman dominating function of $C_{m} \square C_{n}$, and we write $V_{i}^{f}=\left\{v \in V\left(C_{m} \square C_{n}\right) \mid f(v)=i\right\}$ for $i \in\{0,1,2,3\}$. When no confusion arise, we simply write $V_{i}^{f}$ as $V_{i}$. We use $f(i, j)$ to denote the value $f(v)$ for $v=(i, j) \in V\left(C_{m} \square C_{n}\right)$. Let $x_{i}$ be the weight of $\mathscr{C}^{i}$, i.e., $x_{i}=\left\{\sum_{x \in \mathscr{C}^{i}} f(x)\right\}$.

Theorem 3. Let $m, n \geq 1$. Then, the Cartesian product graphs $C_{5 m} \square C_{5 n}$ are double Roman.

Proof. The lower bound follows from Proposition 4. Let $V\left(C_{5 m} \square C_{5 n}\right)=\left\{v_{i j}: 0 \leq i \leq 5 m-1,0 \leq j \leq 5 n-1\right\}$,

$V_{1}=V_{2}=\varnothing, \quad V_{3}=\left\{v_{(5 i)(5 j+2)}, \quad v_{(5 i+1)(5 j)}, \quad v_{(5 i+2)(5 j+3)}\right.$, $\left.v_{(5 i+3)(5 j+1)}, \quad v_{(5 i+4)(5 j+4)}, \quad 0 \leq i \leq m-1, \quad 0 \leq j \leq n-1\right\}$, and $V_{0}=N\left(V_{3}\right)$. Then, $N\left[V_{3}\right]=V\left(C_{5 m} \square C_{5 n}\right)$ and so $f=\left(V_{0}, V_{1}, V_{2}, V_{3}\right)$ is a DRDF of $C_{5 m} \square C_{5 n}$ with weight $15 m n$ and we have $\gamma_{d R}\left(C_{5 m} \square C_{5 n}\right) \leq 15 m n$. Since $\gamma\left(C_{5 m} \square C_{5 n}\right)=5 m n$, we have $C_{5 m} \square C_{5 n}$ is double Roman.

\section{Trees $T$ with $\gamma_{d R}(T)==\gamma_{R}(T)+k$}

Theorem 4. If $T$ is a tree, then $\gamma_{R}(T)+1=\gamma_{d R}(T)$ if and only if $T$ is a star $K_{1, s}$ for $s \geq 1$.

\section{Proof}

$(\Leftarrow)$ If $T$ is a star $K_{1, s}$ for $s \geq 1$, it is clear that $\gamma_{d R}(T)=3$ and $\gamma_{R}(T)=2$, and the theorem holds.

$(\Rightarrow)$ By Proposition 3, we have $\left|V_{3}\right| \leq 1$. If $\left|V_{3}\right|=1$, by Proposition 5, we have $T$ is a star $K_{1, s}$ for some $s \geq 1$. If $\left|V_{2}\right|=0$, then each vertex in $T$ is assigned 2 or 0 . Since $T$ is a tree, $T$ has a least two leaves $v$ and $w$ and $f(v)=f(w)=2$. If $v$ and $w$ are adjacent to the same vertex $x$, then we can obtain a DRDF of $T$ with fewer weight by changing $f(x)$ to 3 and $f(v)$ and $f(w)$ to 0 and obtaining a contradiction. If $v$ and $w$ are adjacent to different vertices, we consider a function $f^{\prime}$ with $f^{\prime}(w)=1, \quad f^{\prime}(v)=1$, and $f^{\prime}(x)=f(x)$ for any $x \in V(T)-\{v, w\}$. Then, $f^{\prime}$ is an RDF of $T$ with weight $\gamma_{d R}(T)-2$. So $\gamma_{R}(T) \leq \gamma_{d R}(T)-2$, a contradiction.

For a positive integer $t$, a wounded spider is a star $K_{1, t}$ with at most $t-1$ of its edges subdivided. In a wounded spider, a vertex of degree $t$ will be called the head vertex, and the vertices at distance two from the head vertex will be the foot vertices.

Theorem 5. If $T$ is a tree, then $\gamma_{R}(T)+2=\gamma_{d R}(T)$ if and only if $T$ is a wounded spider with only one foot or $T$ is obtained by adding an edge between two stars $K_{1, s}$ and $K_{1, t}$ for $s, t \geq 2$.
Proof

$(\Leftarrow)$ If $T$ is a wounded spider with only one foot, it is clear that $\gamma_{d R}(T)=5$ and $\gamma_{R}(T)=3$, and the theorem holds. If $T$ is a tree obtained by adding an edge between two stars $K_{1, s}$ and $K_{1, t}$ for $s, t \geq 2$, then $\gamma_{d R}(T)=6$ and $\gamma_{R}(T)=4$, and the theorem holds.

$(\Rightarrow)$ By Proposition 3, we have $\left|V_{3}\right| \leq 2$. If $\left|V_{3}\right|=2$, let $V_{3}=\{v, w\}$ and $H=G[V(G)-N[w]-N[v]]$. Similar to the proof of Theorem 5, we have $H$ as empty. Otherwise, there exists a vertex $u$ in $H$ assigned with 2 . Now, we change the function values of $v, w, u$ from $3,3,2$ to $2,2,1$, respectively, and obtain an RDF of $T$ with weight $\gamma_{d R}(T)-3$, a contradiction. In this case, $T$ is a tree obtained by adding an edge between two stars $K_{1, s}$ and $K_{1, t}$ for $s, t \geq 2$. If $\left|V_{3}\right|=1$, let $V_{3}=\{v\}$ and $H=G[V(G)-N[v]]$. Then, $H$ has at most one connected component. Otherwise, we can make a vertex in each connected component to change the function values including the vertex $v$ to obtain an RDF of $T$ with weight $\gamma_{d R}(T)-3$. Since $H$ contains no vertex assigned with 3 , then $H$ is not a star with at least two leaves. We claim that the leaves of $H$ are at most two. Otherwise, we change the function values of $v$ and choose two leaves to obtain an RDF of $T$ with weight $\gamma_{d R}(T)-3$. Therefore, $H$ is a path on at least four vertices. In this case, we can obtain an RDF of $T$ with weight at most $\gamma_{d R}(T)-3$, a contradiction. Therefore, $T$ is a wounded spider with only one foot.

\section{Data Availability}

No data were used to support this study.

\section{Conflicts of Interest}

The authors declare that there are no conflicts of interest regarding the publication of this paper.

\section{Acknowledgments}

This work was supported by Sichuan Science and Technology Program under grant 2018ZR0265, Sichuan Military and Civilian Integration Strategy Research Center under grant JMRH-1818, and Sichuan Provincial Department of Education (Key Project) under grant 18ZA0118.

\section{References}

[1] M. R. Garey and D. S. Johnson, Computers and Intractability: A Guide to the Theory of NP-Completeness, W. H. Freeman, San Francisco, CA, USA, 1979.

[2] O. Ore, Theory of Graphs, American Mathematical Society, Providence, RI, USA, 1967.

[3] T. W. Haynes, S. T. Hedetniemi, and P. J. Slater, Fundamentals of Domination in Graphs, Marcel Dekker, NewYork, NY, USA, 1998.

[4] X. Zhang, Z. Li, H. Jiang, and Z. Shao, "Double roman domination in trees," Information Processing Letters, vol. 134, pp. 31-34, 2018. 
[5] E. Zhu and Z. Shao, "Extremal problems on weak roman domination number," Information Processing Letters, vol. 138, pp. 12-18, 2018.

[6] Z. Shao, P. Wu, H. Jiang, Z. Li, J. Žerovnik, and X. Zhang, "Discharging approach for double roman domination in graphs," IEEE Access, vol. 6, pp. 63345-63351, 2018.

[7] Z. Shao, J. Amjadi, S. M. Sheikholeslami, and M. Valinavaz, "On the total double roman domination," IEEE Access, vol. 7, pp. 52035-52041, 2019.

[8] J.-B. Liu, C. Wang, S. Wang, and B. Wei, "Zagreb indices and multiplicative zagreb indices of Eulerian graphs," Bulletin of the Malaysian Mathematical Sciences Society, vol. 42, no. 1, pp. 67-78, 2019.

[9] J.-B. Liu, X.-F. Pan, F.-T. Hu, and F.-F. Hu, "Asymptotic Laplacian-energy-like invariant of lattices," Applied Mathematics and Computation, vol. 253, pp. 205-214, 2015.

[10] J.-B. Liu and X.-F. Pan, "Minimizing Kirchhoff index among graphs with a given vertex bipartiteness," Applied Mathematics and Computation, vol. 291, pp. 84-88, 2016.

[11] H. Yang, P. Wu, S. Nazari-Moghaddam et al., "Bounds for signed double roman $k$-domination in trees," RAIRO-Operations Research, vol. 53, no. 2, pp. 627-643, 2019.

[12] R. A. Beeler, T. W. Haynes, and S. T. Hedetniemi, "Double roman domination," Discrete Applied Mathematics, vol. 211, pp. 23-29, 2016.

[13] R. Hammack, W. Imrich, and S. Klavžar, Handbook of Product Graphs, CRC Press, Boca Raton, FL, USA, Second edition, 2011. 